\title{
Early Detection of Colorectal Cancer and Population Screening Tests
}

\author{
Christos Lionis and Elena Petelos \\ Clinic of Social and Family Medicine, \\ Faculty of Medicine, University of Crete, \\ Greece
}

\section{Introduction}

Issues of early detection of colorectal cancer with reference to the value of screening programmes and the role of the primary care practitioner

Colorectal cancer (CRC) is the most common newly-diagnosed cancer, one of the leading causes of illness and death in the Western world, and the second most common cause of cancer morbidity in Europe. Yet, CRC is a preventable disease and, if detected early, highly treatable. Early detection and prevention are health care strategies of critical importance for the reduction of CRC morbidity and mortality. In a number of countries, screening programmes have been implemented on nationwide scale since the 1960s for other forms of cancer. The early detection of cancer increases the likelihood of successful outcomes, but in order to have early detection, education and training promoting early diagnosis and resulting in increased screening, participation is needed. Additionally, the effectiveness of screening can be measured by the reduction on mortality, but it greatly depends upon tangible and sometimes intangible factors, contingent on setting and target population; it is essential, for example, to identify and screen the appropriate target population and to overcome implementation and uptake barriers. All of these issues, with emphasis on obstacles encountered at the level of general and family practice are highlighted in a recent editorial in Family Practice (Lionis and Petelos, 2011).

Although the screening is performed in the context of public health, and for the benefit of the community, the rights and welfare of the individual should also be respected. The role of the General Practitioner/Family Practitioner (GP/FP) and generally of the Primary Care Provider (PCP) is challenging yet instrumental in achieving this balance, as it is at that level screening is initiated (Viguier et al, 2011). The involvement and the role of GPs and PCPs in convincing patients to participate and initiate CRC screening should be further explored and elucidated, as it is of key importance in cultural and organisational context and health policy issues (Sarfaty, 2006). CRC screening of asymptomatic population groups is currently recommended in the USA and many European countries, and a number of pilot and nationwide programmes have been developed for this purpose. More specifically, mass screening programmes are currently established in 13 of 39 European countries (Pox et al, 2007; Manfredi et al, 2011) with feasibility studies undertaken as pilot actions in many more. 
Although many of these screening programmes, both opportunistic and population-based are already implemented, and screening and early detection of adenomatous polyps has been shown to be effective in the reduction of CRC morbidity and mortality, the rate of screening participation remains low in many population groups at risk for the disease. Good news have recently arrived from across the Atlantic, where decision analysis tools were employed to inform recommendation updates and "microsimulation modelling demonstrated that declines in CRC death rates are consistent with a relatively large contribution from screening" (Edwards et al, 2010), nevertheless, similar efforts are lacking in some European countries, an issue that is given its due importance in this chapter. The success in the US can be attributed to the efforts of international organisations and national task forces, as they have resulted in a level of high awareness of CRC screening among US primary care providers (PCPs) in the US (Klabunde et al, 2003; Levin et al, 2008), but also in certain European countries. However, there is a variation in the evidence that explains the low rate of CRC screening, especially in younger patients (Walsh et al, 2009), while, few physicians recommend screening for the majority of their patients (McGregor et al, 2004). Compounding this effect is evidence that close to a quarter of physicians report not following national screening guidelines, and only half reported the adoption of recommendations that was consistent with the guidelines (Meissner at al, 2006), another key issue that requires special attention.

Additionally, very few PCPs use chart reminders or outreach programmes to contact patient populations most likely to benefit from screening (Klabunde et al, 2009). There is limited research focusing on obstacles and barriers, and the role of the physician-patient relationship plays in determining participation in screening programmes, especially when it comes to ethnic and culturally diverse groups (Lionis and Petelos, 2011). The importance of culturally relevant strategies for designing and implementing screening programmes has been already highlighted (Tu et al, 2006). Additionally, the role of socioeconomic disparities in CRC screening has been highlighted and documented (Meissner et al, 2011) if not explored in detail (Aubin-Auger et al, 2011), thus indicating a need for a close collaboration between medical and social care scientists in order to improve the requisite understanding for increased compliance to CRC screening recommendations. To compound the increasing complexity of national guidelines and the sensitivity of implementing them to culturally and linguistically varied patients, support through interventions focusing on organizational changes and further education and training for PCPs on early diagnosis, prevention and health promotion is needed. These are all issues that this chapter attempts to address. All of these factors are relevant for and have an impact on the ongoing debate about the role of GPs/FPs and PCPs, as well as the contribution these have on the effective implementation of screening programmes, opportunistic and population-based.

From all of the above one can surmise that the early detection of CRC is an issue of complexity requiring clear messages to increase the awareness and performance of the health care actors. This is another objective of the present chapter. Thus, the particular aims of this chapter are: (a) to provide information about the recommendations issued by certain large national and international organizations, including those issued by the U.S. Preventive Services Task Force (US PSTF) on the use of the available screening tests for the early detection of CRC and adenomas for average-risk subjects, (b) to critically review the role of clinical physicians and mainly PCPs in the early detection of CRC, (c) to explore issues with an impact on CRC screening, and, finally, (d) to highlight some quality issues relevant to CRC screening and relevant guidelines for quality assurance mechanisms in the relevant 
processes. The chapter starts with concepts and definitions, proceeds with the recommended screening tests and concludes by outlining main points of interest and corresponding recommended tasks and actions for PCPs, for the purpose of increasing uptake and facilitating implementation of CRC screening programmes.

\section{Concepts and definitions}

Population screening is the systematic application of a suitable test with the aim of identifying individuals at a risk of a specific condition or disorder, but who have not sought medical attention on account of symptoms for that particular condition or disorder, and who can benefit from further investigation or direct preventive action (Wald, 1994). The notion differs from opportunistic screening, and it is a systematic process that includes certain steps from call or recall to screening, feedback of the results and follow-up in welldefined intervals. For population screening, the organised framework in which it takes place provides opportunities for more effective management, quality assurance and evaluation.

In our empirical view, understanding of the notion of screening, population or opportunistic, greatly varies between health care practitioners with the result of adversely impacting the effective implementation of the early detection programmes for CRC. It is for this reason we have decided to provide an extensive review on the existing literature and consensus criteria to define screening, focusing on CRC screening.

As stated by Wilson and Jungner in their seminal paper (Wilson and Jungner, 1968) "the central idea of early disease detection and treatment is essentially simple. However, the path to its successful achievement (on the one hand bringing to treatment those with previously undetected disease, and, on the other, avoiding harm to those persons not in need of treatment) is far from simple though sometimes it may appear deceptively easy". On the basis of whether early detection is possible at an early stage of the disease and taking into consideration whether an appropriate treatment is available, they attempted to formulate criteria that could help guide the selection of conditions and population groups suitable for screening. They also noted case-finding differences, depending on whether it is performed by a public health agency or by a general practitioner, and, almost four decades ago, emphasised the aspect of cost by underlining the importance of assessing effectiveness not only from an individual, but also from a public health perspective.

The fast pace of genetic research and the advent of new therapies has resulted in the generation of many other lists of screening criteria; most of them based to a greater or lesser degree on the Wilson-Jungner criteria. Additionally, even when consensus at the national or regional level is reached on which set of criteria to apply, there are other social, ethical and even logistical considerations to be examined. More recent trends on patient-centric and evidence-based health care, as well as cost-effectiveness and quality assurance, have resulted through a series of consultations to the modified Wilson and Jungner criteria (Andermann et al, 2008). In these amended criteria, opportunistic screening, essentially casefinding performed outside a framework of an organised programme as the one required to ensure such criteria are met, is, therefore, not a valid alternative; additionally to being less efficient it is also more costly, and, most importantly, quality assurance mechanisms cannot be embedded in a standardised fashion in such a process.

The definition of an "organised" screening programme according to the International Agency for Research on Cancer (IARC) includes: 1) an explicit policy with specified age categories, method and interval for screening; 2) a defined target population; 3) a 
management team responsible for implementation; 4) a health-care team for decisions and care; 5) a quality assurance structure; and 6) a method for identifying cancer occurrence and death in the population (IARC 2005). Such organised population-based screening programmes have a predefined specific population, according to epidemiological data and on the basis of target age and geographical area, and during all the stages, from the invitation of the eligible individuals to the assessment procedures following testing, a specific protocol is followed. As mentioned, quality aspects of the process can be better addressed, as for example during follow-up (Miles et al, 2004). Furthermore, such screening programmes usually do not incur any costs for the participants.

It is important to have also a concrete idea regarding more abstract terms determining the usefulness and, even, the effectiveness of screening tests, and to keep such definitions in mind. Although these terms have been widely used, there is also great variation of their understanding and usage in clinical decision-making. For example, the ability of a measure or test to predict a subsequent event is a form of validity. On the basis of such a criterion we determine the predictive value of a test. The positive predictive value (PPV) in terms of detection through FOBT screening is defined as "the percentage of people with detection of at least one lesion/adenoma/advanced adenoma/cancer at follow-up colorectal screening among those with positive tests who have attended follow-up colorectal screening" [Adapted from the European guidelines for quality assurance in colorectal cancer screening and diagnosis. 2011. European Commission, Directorate General for Health and Consumers, EAHC - Executive Agency for Health and Consumers, World Health Organisation], whereas a positive test is, effectively, an abnormal result leading to further investigation (i.e. colonoscopy) or the removal of a lesion, for example, according to the protocol of the organized screening programme. By alluding to a false-positive result, we effectively mean that although the test indicated disease is present this is not the case. In a true-positive test, the result is correct and the disease is really present. Similarly a false-negative test indicates a disease-free subject has been tested, but the disease is present and might remain undetected if there is no further testing, symptoms, etc., whereas a true-negative test indicates a disease-free subject has been tested. Prevalence of the disease affects not only the positive predictive value of a screening test but also its negative predictive value, i.e. the probability that the person subjected to the screening test is truly free from the disease when a negative (normal) test result is obtained.

Two concepts often discussed in relation to true- and false- positive and negative results are sensitivity and specificity and both terms have an important impact on the PCP decisions on which of the available tests for the early detection of CRC should be recommended. The sensitivity refers to the number of cases the test can identify or in more simple words the probability of one diagnostic test telling the truth when the disease exists. It gives us a certainty that true positives will not be missed. Specificity refers to the accuracy of the finding or in simple words the probability in telling the truth when the disease is absent. Ideally, a test should be both highly sensitive and specific. To that direction, the US Preventive Services Task Force and the Institute of Medicine (IoM) recommend the fecal occult blood test (FOBT) test, and more specifically the guaiac test (gFOBT) for screening programmes. Nevertheless, when used on its own, it has relatively low sensitivity, whereas the a combination with a more sensitive test, such as the fecal immunochemical test (FIT) could help to render screening programmes more effective (Allison, et al, 2007). Another interesting consideration, especially given the public health context of mass screening, is cost-effectiveness and how it correlates to specificity and sensitivity. Although uncertainty 
remains, the assessment of a screening program based on FIT for a one-year period in France seemed to be the most cost-effective approach (Hassan, et al, 2011). More research is necessary, as for example indicating what the best cut-off levels for colonoscopy referral are, without compromising sensitivity, to determine optimal public health approaches.

\section{Screening tests, guidelines for CRC and the importance of early detection}

As previously mentioned, CRC can be curable when diagnosed at an early stage. Also, CRC mostly develops from colorectal non-malignant precursor lesions, thus, rendering it a preventable disease through the removal of premalignant lesions. Systematic early detection and removal at the "adenoma-phase" can prevent the occurrence of CRC and markedly decrease overall population incidence (Winawer et al, 1993), as human colon carcinogenesis progresses to the carcinoma pathway via the dysplasia-adenoma phase.

The readers of this chapter are aware from previous chapters of this book that there are various tumour staging systems, the ones mainly used in Europe being Duke's classification and TNM (Tumour, Node, Metastasis) classification of malignant tumours, introduced by the Union Internationale Contra le Cancer (UICC) and the American Joint Committee on Cancer (AJCC). Despite the fact TNM yields greater information, there are several major issues due to the reclassification of the system. Most importantly, there seems to be great disparity between the therapeutic decision-making and the TNM staging, with multiple TNM staging versions being used in different countries and great variance in reporting. It has been argued that changes should only occur after extensive discussion within the scientific community (Quirke et al, 2010), and it is essential to note that the reporting on a nationwide scale for any given CRC screening programme should be performed on the basis of the same staging system. Lesion reporting within the frame of the screening programme should be standardized to allow for better evaluation and reporting, and, consequently, improved outcomes. TNM stages and version, frequency of CRC and distribution of TNM stages should be reported along with the presence of non-neoplastic lesions. According to the report of the EU on CRC quality guidelines (European Commission, Directorate General for Health and Consumers, EAHC - Executive Agency for Health and Consumers, World Health Organisation, 2011), without explicit criteria for the diagnosis and staging of early adenocarcinoma unnecessary radical resection would result in severe overtreatment, raising the morbidity and mortality in the context of the programmes.

Various screening technologies are currently available, from the more established Guaiac faecal occult blood tests (gFOBT) and immunochemical FOBT to sigmoidoscopy and colonoscopy, as well as combinations (i.e. combined FOBT with sigmoidoscopy) to the new screening technologies, as CT colonography, stool DNA and capsule endoscopy. Early reports during the previous decade suggested that biennial screening by FOBT reduces CRC mortality, as for example in the French (Faivre et al, 2004) and Danish populations (Jørgensen et al, 2002). This fact lead WHO (World Health Organization) and OMED (World Organization for Digestive Endoscopy) to suggest a choice of FOBT that should take into account dietary compliance to recommendations, but also colonoscopy resources (Young et al, 2002). Prior to any recommendation for screening tests for CRC, the PCPs should be able to recognise whether the individual visiting the practice/office is at average, increased or at high risk for CRC. In other words, to be able to identify whether the particular individual truly belongs to the target population of the screening programme or -should there not be one available- whether there is reason for referral in the context of opportunistic screening. 
In Australia, the Department of Health and Aging has issued clinical practice guidelines for the prevention, early detection and management, and national population-based screening programs are in place for various cancers, from breast cervix and to bowel (CRC) (Australian DoHA, 2011). Also, the National Bowel Cancer Screening Program Register plays an important role in the programme, as it assists participants through the screening pathway, allows for reminders and follow-ups without taxing local resources and GPs. There are online tools and decision-aids available to GPs and information in twenty languages targeted at patients. Pre-invitation, invitation, follow-up letters, FOBT kit instructions and an information booklet are all provided in all of these languages and are also available online. Additionally, a qualitative evaluation of opinions, attitudes and behaviours influencing CRC were examined in the pilot phase of the national screening programme and the report was published and integrated in future planning [A Qualitative Evaluation of Opinions, Attitudes and Behaviours Influencing the Bowel Cancer Screening Pilot Program: Final Report August 2005]. Most interestingly, the invitation is sent directly to the candidate participant and it is not necessary to nominate a physician in the forms submitted, although participants are encouraged to nominate a doctor in the context of follow-up if the FOBT is positive:

a. If no doctor is nominated, the FOBT results will only be sent to the participant.

b. If a doctor is nominated, the results of the FOBT will be sent to the participant and their doctor.

c. If the FOBT result is positive it is explained that it will be necessary to discuss the result with a doctor.

The American Cancer Society (ACS) and the National Colorectal Cancer (NCC) in cooperation with the Thomas Jefferson University have edited a Primary Care Clinician's Evidence-Based Toolbox and Guide (Sarfaty, 2008). According to this guide, an individual is at an average risk when s/he has no first-degree relatives with a history of either CRC or adenomatous polyps and no illness or past health problems have been reported (Sarfaty, 2008). For individuals at average risk, GPs are recommended to initially take a medical history, including age, symptoms, family medical history, and also individual history with a focus on bowel diseases and dietary habits, and to perform a clinical examination including a digital rectal examination. Also, various CRC screening guidelines and recommendations have been issued, both by national and international organisations and institutions. In 2008, a joint effort of the ACS and the American Gastroenterology Association was released regarding certain modalities including stool tests, flexible sigmoidoscopy (FS), colonoscopy (CS), double-contrast barium enema (DCBE), computer tomography, colonography (CTC) (McFarland, et al 2008). Those joint guidelines also stressed the importance of prevention of CRC important tasks for PCPs. The US Preventive Services Task Force (US PSTF) recommends routine asymptomatic screening for three cancer sites, including that of breast, CRC and cervix, mainly because they are asymptomatic to a high degree in early staging, have a high 5-year survival rate when the cancer is localised, and as there is a strong evidence on the screening effectiveness (Cardarelli, 2010).

In terms of a recommended start and stop age for screening, the ACS has issued guidelines for the early detection of CRC and polyps with recommended screening beginning at age 50 for both men and women (ACS, 2011). The US PSTF recommends a screening for averagerisk men and women 50 years of age and older, with colonoscopy every 10 years, flexible sigmoidoscopy or DCBE every five years and faecal occult blood test every year (U.S. Preventive Services Task Force, 2011). In a supporting document, this Task Force 
summarises its recommendations and recommends screening for CRC using an FOBT, sigmoidoscopy, or colonoscopy in adults beginning at the age of 50 years and continuing until the age of 75 (Grade: A Recommendation). However, US PSTF recommends against screening for CRC in adults over 85 (Grade: D Recommendation), while it concludes that the evidence is insufficient to assess the benefits of CT colonography and faecal DNA testing for $\mathrm{CRC}$ as screening modalities. Judging the benefits against harms, the American Task Force discusses among the benefits of the less invasive CRS screening the number of colonoscopies that may be reduced. However, it recommends that for any positive test there is a follow-up with colonoscopy. The Task Force Recommendation statement underlines that the benefits of CRC detection and early intervention decline at the age of 75 years, thus it leaves the decision for a routine screening at individual level. There is, as described, a lot of information, but slightly conflicting evidence and advice. Nevertheless, participation of the $50-75$ years age group increased by $13.1 \%$ reaching $65.4 \%$, whereas a significant CRC incidence decline was noted in 35 states and mortality declined in 49 states and DC (CDC, 2011). Further efforts are currently being made in the field of patient engagement and patient-reported outcomes, and in the context of comparative effectiveness research (PCORI, 2011).

In the United Kingdom, Cancer Research UK underlines the importance of screening for the reduction of CRC mortality and has elaborated upon the role of FOBT and flexible sigmoidoscopy (Cancer Research UK, 2011). This institute refers to evidence provided by four RCTs where the use of FOBT every two years reduced CRC mortality by $15 \%$ to $18 \%$ in people aged 45-74 years. Centralised systems, such as the Australian and, to a certain extent, the UK system, remove pressure from the individual GP and the organisational capacity at practice level, but could potentially result in a loss of involvement and a lowered feeling of responsibility.

An individual is at increased risk when s/he has a personal and family history of CRC or adenomatous polyps but without reporting any of the high-risk familiar syndromes. Those hereditary syndromes include: the hereditary non-polyposis CRC (HNPCC), the familiar adenomatous polyposis (FPP) and the attenuated PAP (APAP). In this group, the clinical physicians and the PCP should change their strategy from screening to regular surveillance, and the tests that primarily detect cancer should be replaced by more sensitive diagnostic approaches and particularly colonoscopy, which should start at age 40 or younger (Sarfaty, 2008). The National Institute for Health and Clinical Excellence (UK) has recently published a new guideline on colonoscopic surveillance for the prevention of people with ulcerative colitis, Crohn's disease or adenomas (NICE, 2011). At the third category where the probability of developing CRC is high, the PCPs should be more cautious when recommending screening and surveillance. A family history of an adenomatous polypus or CRC in a relative under the age of 50 is suggesting a high probability of the presence of any of the above high-risk hereditary syndromes and the clinical physician requires genetic testing; a close collaboration with hospital specialists at a centre with expertise should be established (Sarfaty, 2008).

In Europe, the high degree of heterogeneity in health care systems, policy, roles, screening programme resources and very different values in local and regional settings had previously created a rather fragmented picture. There are ongoing efforts toward harmonisation, for example, recently developed guidelines (2011) in an effort under the auspices of the European Commission, focus on quality assurance and provide clear and concise information to facilitate decision-making at the GP/FP and PCP levels. As illustrated by 
Table 1, an evidenced-based brief overview of various conventional screening methods is given, although new technologies resulting in more modern forms of screening are not assessed for lack of evidence; some information regarding cost-effectiveness is provided along with the recommendations and examined in more details in the report (European Commission, Directorate General for Health and Consumers, EAHC - Executive Agency for Health and Consumers, World Health Organisation, 2011)

Finally, it is important to underline that early detection is directly dependent on acceptance of the screening test by both provider and patient, as well as the uptake of the screening programme. For example, early versions of stool DNA (sDNA) testing lacked the requisite sensitivity and markers, but improved sDNA tests are now available. It is important to understand patient preferences regarding screening options for selecting the right tool for a given population; for example, whether a non-invasive test is preferred to colonoscopy or whether accuracy is considered much more important than discomfort. In a study by Schroy et al, (2002), those preferring colonoscopy to sDNA or FOBT rated accuracy as the most important factor, whereas those rating concerns about discomfort or frequency of testing as the most important parameter preferred sDNA. Most subjects preferred a shared (54\%) or patient-dominant (34\%) decision-making process.

As previously highlighted, removal of all adenomas, without accurately distinguishing between those which will become malignant and those which will not, will effectively result in excessive overtreatment, and it is for this reason that newer screening tests, such as the sDNA, focusing on genomic changes affecting associated biological and metabolic processes should not be overlooked as options necessitating further research -particularly because of their potential to avoid iatrogenic care, but also because they might better reflect patient preferences for certain population groups (Sillars-Hardebol, et al, 2012).

[Adapted from the European guidelines for quality assurance in colorectal cancer screening and diagnosis. 2011. European Commission, Directorate General for Health and Consumers, EAHC - Executive Agency for Health and Consumers, World Health Organisation.]

Guaiac FOBT

There is good evidence that invitation to screening with FOBT using the guaiac test reduces mortality from colorectal cancer (CRC) by approximately $15 \%$ in average risk populations of appropriate age

RCTs have only investigated annual and biennial screening with guaiac FOBT (gFOBT) (II). To ensure effectiveness of gFOBT screening, the screening interval in a national screening programme should not exceed two years

Circumstantial evidence suggests that mortality reduction from gFOBT is similar in different age ranges between 45 and 80 years. The age range for a national screening programme should at least include 60 to 64 years in which CRC incidence and mortality are high and life expectancy is still considerable. From there the age range could be expanded to include younger and older individuals, taking into account the balance between risk and benefit and the available resources

Immunochemical FOBT

There is reasonable evidence from an RCT that iFOBT screening reduces rectal cancer mortality, and from case control studies that it reduces overall CRC mortality; Additional 
evidence indicates that iFOBT is superior to gFOBT with respect to detection rate and positive predictive value for adenomas and cancer

Given the lack of additional evidence, the interval for iFOBT screening can best be set at that of gFOBT, and should not exceed three years

In the absence of additional evidence, the age range for a screening programme with iFOBT can be based on the limited evidence for the optimal age range in gFOBT trials

Sigmoidoscopy

There is reasonable evidence from one large RCT that flexible sigmoidoscopy (FS) screening reduces CRC incidence and mortality if performed in an organised screening programme with careful monitoring of the quality and systematic evaluation of the outcomes, adverse effects and costs

The available evidence suggests that the optimal interval for FS screening should not be less than 10 years and may even be extended to 20 years

There is limited evidence suggesting that the best age range for FS screening should be between 55 and 64 years. After age 74, average-risk FS screening should be discontinued, given the increasing co-morbidity in this age range

Colonoscopy

Limited evidence exists on the efficacy of colonoscopy screening in reducing CRC incidence and mortality. However, recent studies suggest that colonoscopy screening might not be as effective in the right colon as in other segments of the colorectum

Limited available evidence suggests that the optimal interval for colonoscopy screening should not be less than 10 years and may even extend up to 20 years

Indirect evidence suggests that the prevalence of neoplastic lesions in the population below 50 years of age is too low to justify colonoscopic screening, while in the elderly population (75 years and above) lack of benefit could be a major issue. The optimal age for a single colonoscopy appears to be around 55 years. Average risk colonoscopy screening should not be performed before age 50 and should be discontinued after age 74

Combination of FOBT and sigmoidoscopy

The impact on CRC incidence and mortality of combining sigmoidoscopy screening with annual or biennial FOBT has not yet been evaluated in trials. There is currently no evidence for extra benefit from adding a once-only FOBT to sigmoidoscopy screening

New screening technologies under evaluation

There currently is no evidence on the effect new screening tests under evaluation on CRC incidence and mortality. New screening technologies such as CT colonography, stool DNA testing and capsule endoscopy should therefore not be used for screening the average-risk population

Cost-effectiveness

Costs per life-year gained for both FOBT and endoscopy screening strategies are well below the commonly-used threshold of US\$ 50000 per life-year gained (LYG)

There is some evidence that iFOBT is a cost-effective alternative to gFOBT

Available studies differ with respect to what screening strategies are most cost-effective.

No recommendation of one screening strategy over the others can be made based on the available evidence of cost-effectiveness

Table 1. Recommendations and conclusions 
Finally, the concepts of colonoscopic surveillance and screening for recurrent CRC should receive attention by PCPs. The adenomatous precursors of CRC are present in over $30 \%$ of individuals over 55 (Eide, 1991), placing them at higher risk of developing CRC, but the removal of these lesions reduces risk to that of the general population (Citarda et al, 2001). Recurrent CRC, as for example following resection, also necessitates an intensive surveillance programme, as the detection at an asymptomatic stage can result in survival benefit (Renehan, et al, 2002). This means that surveillance and follow-up programmes should also be combined or evaluated along with a screening programme.

\section{Primary care and CRC: Tasks and steps for screening implementation in primary care}

One of the most important factors for the effective implementation of a CRC screening programme is the involvement of a $\mathrm{PCP}$, particularly of the GP or the FP, in convincing targeted individuals to participate and to initiate the screening. The PCPs have multiple and varying tasks, more specifically to (Sarfaty, 2008):

1. Assess the risk of developing CRC and increase the risk awareness, as described above.

2. Discuss options with patients/individuals and effectively engage in shared decisionmaking (SDM) - this would ensure patient perspectives and preferences are consistent to decisions made.

3. Convince to participate - this task requires communication and consultation skills, as well as an established continuity of care.

4. Implement the initial tests: those primarily used to detect cancer, including the annual Guaiac-based occult blood test (gFOBT), the annual faecal immunochemical test (FIT), or stool DNA test (sDNA).

5. Consider and assess the available screening resources and capacity: it is an important task for PCPs, who should be aware of the available resources in their district or health region capacity, as well as patient limitations (e.g. socioeconomic, mobility, etc.), to determine the optimal referral pathway for the test(s) that detect adenomatous polyps and CRC (FS, CS, DCBE, CRC).

6. To make the necessary arrangement to complete the CRC screening.

One of the most challenging issues that the PCPs encounter is to convince the average risk individual to use a simple and inexpensive test to initially detect if any hidden blood is present in stools, constituting a strong indication of the presence of an adenomatous polyp or CRC. To achieve it, an effective doctor-patient communication should be established, and the purpose of the GPs/PCPs might also need to be re-assessed by further education or training on early diagnosis, prevention and promotion. The role of a multidisciplinary team is also essential. FOB Testing serves this role, although it has received criticism because of the lack of specificity, particularly when the test is dehydrated, and because of the subsequent increase of the associated costs of screening programmes (WHO, Rudy and Zdon, 2000). FIT, also, fulfils this purpose; it is a simple procedure: the stool sample is collected by the individual/patient at home, and the completed test is sent to a laboratory or to the PCPs office. Usually two samples from different bowel movements are required and the instructions on sampling procedures on how the water sample should be transferred by the brush onto the test card are clear and readily understood. 
Another important task for PCPs and other practitioners is to educate their patients/clients to contact the PHC services when some warning signs are experienced and among them are (Rudy and Zdon, 2000):

- Hematochezia

- Melaena

- Anaemia resulting from occult blood loss

- Change in bowel habits

Prior to the decision of the PCP to refer the subjects to either CS/FT or CT should be explored the access to that screening method and consider the existing diagnostic capacity resources (Sarfaty and Wender, 2007).

Finally, another essential consideration in the PCP decision to implement screening tests for early detection of CRC is that of quality of life. Quality of life in evidence-based medicine should always reflect the preferences of patients, as patient-centeredness is its cornerstone. Despite the fact everyone values particular aspects of life differently, all aspects of life that may be affected adversely or in a beneficial manner by aspects of health and illness should be taken into consideration. For screening programmes, it is important to understand the cultural context in which it is performed or is to be performed and to ensure the values of the patients are taken into consideration when determining and/or assessing outcomes.

\section{Obstacles to implementing CRC screening in primary care}

\section{Obstacles in primary care}

As mentioned above, the CRC screening rate increase does not seem to apply in many countries and regions and the associated obstacles and barriers that have already been reported in the literature (Lionis and Petelos, 2011) could be classified as follows:

- Obstacles at doctor level: Obstacles reported by the GPs were relevant to the difficulties in being convinced especially when signs and symptoms were lacking. There was, in other words, confusion in addressing difficulties stemming from conflict between personal experiences and public health implications (Aubin-Auger et al, 2011). Also, there is research indicating that even in countries with established screening programmes only $50 \%$ of the GPs considered themselves to be sufficiently trained, as for example in France (Viguier et al, 2011).

- Obstacles at patient level: Researchers examined obstacles at patient level and how these were linked to the physician-patient interaction and communication. For example, cancer screening did not fall in with the perception of some patients regarding health care, and they failed to identify benefits outside the context of familiar high-risk groups. Potentially inadvertently reflecting specificity and sensitivity issues, participants were afraid of poor technical skills, and taking ownership of the risk for performing the test, resulting in false positive or false negative results (Aubin-Auger et al. 2011). Mirroring the high number of GPs who do not feel they are sufficiently trained, patients cited the absence of recommendation as one of the most important reasons for not undergoing screening (Viguier et al, 2011).

- Obstacles at doctor-patient level: GPs and patients agreed the lack of symptoms and lack of familial risk were two of the main reasons for doubting how useful such a test could be, the GPs thought that the patients misunderstood the process and were 
afraid of reactions to false negative results, whereas the patients complained about time, as well as the constipation effect from repeating the test, and did not express fears about such results (Aubin-Auger et al, 2011). Further evidence (Schroy et al, 2011) indicates that screening intentions and test ordering are adversely affected when patient and provider preferences differ. Interestingly, compounding previously reported data (Serra et al, 2008), having a screening habit (e.g. mammography) proved to be a positive factor for women, whereas increased participation was reported for those with a higher educational level, particularly for male patients. Without diminishing the importance of facilitators, a patient having a relative having already performed gFOBT was more likely to accept the test, but friends and family were not identified as obstacles.

\section{Further barriers:}

Cultural and linguistic barriers were also touched upon by these researchers, but not explored in detail; it is highlighted that even the wording a doctor uses has an effect and that further research is necessary (Lionis and Petelos, 2011). There is evidence that by employing culturally and linguistically relevant approaches for FOBT promotion, screening participation increases in target populations of low-income and/or less acculturated minority patients ( $\mathrm{Tu}$ et al, 2006). Indeed, a challenge of equal significance to guideline adherence and compliance in screening is ensuring equity of access to screening. Part of ensuring equity of access is to ensure awareness issues have been addressed for all ethnic and culturally diverse groups. A study of all the patients aged 50-60 registered in general practices for a UK region (West Midlands), with a total number of over eleven thousand respondents, examined factors that contributed positively or negatively on behaviour toward screening (Taskila et al, 2009). People without a screening habit (men), older people, and those with Indian ethnic backgrounds were more likely to have negative attitudes, whereas Black-Caribbean ethnic background people reporting abdominal pain, bleeding or tiredness were more likely to have a positive attitude. This great variation in attitudes indicates that there are different needs to be addressed for increasing awareness and highlight the importance of culturally relevant strategies for designing and implementing screening programmes (Taskila et al, 2009). Evidence amasses from various countries, with a study focusing specifically on FOBT use, along with the subsequent investigation of a positive result (Bampton et al, 2005). Researchers established that both indications for use and follow-up of a positive result varied according to the ethnicity of the GP and independently of the medical training received (Koo et al, 2011). Additionally, it was indicated that the ethnicity of the patient and, similarly to results of other research, associated linguistic and cultural barriers affect screening uptake and was noted that this may adversely affect the health of immigrant populations.

To address all the obstacles and barriers previously mentioned, it is necessary to embrace the perspective of the users of screening programmes, and also to examine screening under the prism of public health perspective. A recently conducted review highlights the need for policy supporting both screening delivery and organisational transformation in a manner that promotes improvement of operational features for preventive services (Senore et al, 2010). The researchers examined recently proposed conceptual frameworks that were aimed at identifying key elements and, thus, potential targets for interventions aiming to improve screening (Cole et al, 2009 and Federici et al, 2005). The models developed conceptualised these potential targets at various levels: the organizational 
context in which health care delivery and provision are taking place, the practice itself, and the structural and operational characteristics of given settings, and also examined the provider and patient levels. The researchers concluded that although a given intervention may be implemented at one or multiple levels, the factors determining uptake and participation are, indeed, correlated with all of these levels in an interconnected and interdependent manner.

In concluding this section, the role of the PCPs is extremely complex, and although research on obstacles, barriers and limitations is starting to create a more robust evidence base, further qualitative and translational research is required to identify best practices and intervention transferability. Additionally, policy measures for the purpose of supporting screening delivery mechanisms are required, and, similarly, policy should aim to facilitate the organisational changes necessary for creating and supporting the operational features of preventive services.

\section{Increasing the CRC screening rate}

Although messages about the effectiveness of CRC screening have been widely available, there are still concerns in terms of both physician involvement and PC user participation in CRC screening. This is not a message that concerns CRC screening per se, but prevention and health promotion activities undertaken by GPs in Europe. There are significant gaps between GP knowledge and practice in Europe, already reported upon (Brotons et al, 2005). Evidence from the literature indicates that less than one third of the PC physicians use chart reminders and $15 \%$ use outreach mechanisms to contact patients needing screening (Klabunde et al, 2009). Investment has been made on efforts and research programs to assess the impact of quality improvement intervention programs. One of them combined diverse components, such as performance activities, delivery system design, electronic medical record tools and patient activation (Ornstein et al, 2010), and reported promising results in the Evidence-Based Toolbox and Guide we currently have (Sarfaty, 2008). Thus, the implementation of educational programmes for PCPs and patients in addition to the development of shared-decision making tools, given the differing perspectives between doctor and patient, seem imperative, as otherwise lack of consensus could adversely impact the CRC screening rate (Schroy et al, 2011).

A page invitation to the health practitioners to avoid certain errors has been made and among them the following:

- To screen for CRC with only a digital rectal exam or with a single sample from a stool blood test

- Recommend screening with colonoscopy at average risk more often than every 10 years or CT colonography, DBCE or flexible sigmoidoscopy more often than five years

A toolkit for a systematic approach in tracking and increasing screening for public health improvement of CRC intervention was prepared for the Agency for Health Care Research and Quality (AHRQ). It delivers tools, process guidelines, tips and evidence of the intervention effectiveness (Harris et al, 2010). It is strongly recommended for PCPs, health care planners and managers. However, the role of PCPs in increasing the CRC screening rate remains a key component. According to the ACS and NCS, the positive impact of its 
advice is well documented, and the magnitude of the doctor's impact is considerable (Sarfaty, 2008).

\section{Designing a national CRC screening programme/framework}

We have seen the importance and potential of CRC screening in detail. The importance of screening taking place in an organised framework for optimal results, as for example in nationwide programmes, has also been examined and noted. As many European countries are still in the process of designing such a programme and many other countries globally are far from implementing such interventions, it is important to see how to best learn from other experiences and how to use lessons already learned to help us morph a flexible and robust model that can be adapted according to regional and local needs to ensure high acceptance, uptake and, indeed, equal access and reduced disparities. Thus, we decided to include in this section some key issues that health planners, health policy makers and public health decision makers should take into account when considering the design and implementation of a CRC screening programme.

Many of the countries in which a nationwide programme is implemented have extensively reported on the outcomes and evaluation of such programmes. Australia has introduced such a programme, but reporting indicated the needs and beliefs of minority groups, as for example indigenous Australians, were not always taken into consideration, with stronger drives, as for example economic benefit at country level, determining the approach undertaken and the strategy selected (Christou and Thompson, 2010).

To start, the need of conducting a feasibility study should be evaluated. According to Bowen et al, (2009) performing a feasibility study may be indicated when "(a) community partnerships need to be established, increased, or sustained, (b) there are few previously published studies or existing data using a specific intervention technique, (c) prior studies of a specific intervention technique in a specific population were not guided by in-depth research or knowledge of the population's socio-cultural health beliefs, by members of diverse research teams, or by researchers familiar with the target population and in partnership with the targeted communities, (d) the population or intervention target has been shown empirically to need unique consideration of the topic, method, or outcome in other research or (e) previous interventions that employed a similar method have not been successful, but improved versions may be successful; or previous interventions had positive outcomes but in different settings than the one of interest". By quickly reviewing these grounds it becomes apparent CRC programmes, independently of whether they are still being designed or already implemented, are prime candidates for qualitative research via feasibility studies. These pilot actions can help elicit patient preferences and elucidate obstacles adversely affecting participation. In terms of results, even small modifications to existing programmes or design can greatly affect outcomes. For example, a sound review being the starting point for deploying such a pilot action, recent systematic reviews of interventions in Australia, indicated that organisational level changes were the most effective in terms of screening behaviour enhancement. It is important to note necessary modifications for increased effectiveness were those that included non-physicians in the screening process (Christie et al, 2008, Wardle et al, 2003, Vernon 1997). Language and literacy barriers are, of course, the most difficult to overcome, as illustrated by the Alaskan and Australian Aboriginal examples, and can only be adequately researched and addressed 
through engagement of local actors and community leaders, strong community orientation and high level of awareness of the PHPs, including most importantly nursing and all other available healthcare personnel -especially in rural or remote areas.

Additionally, elements of the care pathway ought to be assessed in context and real settings, and, especially where pragmatic trials are impossible, difficult or unethical. Participation in and completion of the screening test is not the only necessary part to ensure successful outcomes, as there has to be a follow up with the appropriate diagnostic testing. (Christou and Thompson, 2010).

Screening utilisation is also influenced by behavioural factors and health economics parameters, as well as by the organizational and cultural settings (Senore et al, 2010). A theoretical framework to explain the adoption of health-related behaviours is needed to underpin any given implementation effort. Models conceptualizing elements of the health care provision have been proposed, providing targets for intervention at patient and provider levels (Stone et al, 2002; Bastani et al, 2004, Senore et al, 2010). However, it is important to note that although any given intervention component may act upon more than one levels, screening uptake is interdependent on all these factors (Senore et al, 2010).

Consideration of multidisciplinary teams is also essential. These teams can help direct resources along a predefined, according to the evidence-base, care pathway ensuring effective, efficient and sustainable implementation and, thus, better results. As we have previously discussed, CRC screening is complex and comprises of different stages involving the participation of different health care actors. For example, all abnormal results should be followed-up and after-care service following treatment should be available. Nevertheless, only a small portion of health plans monitor follow-up care (Klabunde et al, 2003). Multidisciplinary participation can help implement interventions that have added value at patient, provider and even public health-health care system level. For example same day follow-up for abnormal FS, offering on-site colonoscopy seems to lead to better compliance (Stern et al, 2000; Senore et al, 2010). Another benefit in the involvement of nursing and clerical staff is the integration of quality indicators and quality assurance mechanisms in delivery processes and their monitoring as part of standardised care delivery without draining on valuable resources.

\section{CRC screening: An issue of quality assurance in modern health care systems}

Quality issues in colorectal cancer screening have been previously discussed in editorials in the journals of Quality in Primary Care and Family Practice (Lionis, 2007, Lionis and Petelos, 2011). These editorials address issues relevant to improvement of uptake of CRC screening with the use of cognitive methods and the translation of the Health Belief Model into education and training programs for health care providers. The authors call for a closer collaboration between medical and social care scientists, and reveals another important challenge that PCPs face: addressing health inequalities in a changing and financially restrained world, where for example, minority groups showcase low adoption rates of preventive measures and screening tests.

As previously mentioned, the Directorate General for Health and Consumers, with tasks funded by the EU Health programme (CRC screening grant No 2005317), led an effort 
aiming to develop EU guidelines on best practice in CRC screening, which resulted in the publication of the first edition of the European guidelines for quality assurance in colorectal cancer screening and diagnosis in February of 2011 (European Commission, Directorate General for Health and Consumers, EAHC - Executive Agency for Health and Consumers, World Health Organisation). The guidelines systematically examine the evidence for efficacy and effectiveness of CRC screening and outline the guiding principles for organising CRC screening programmes. Most importantly, the authors underline the importance of the availability of comprehensive, evidence-based quality assurance guidelines that address all the steps of a screening programme, including invitation, information, surveillance and any other subsequent care, as a key factor to the success of any cancer screening programme. Finally, the authors advocate the widespread application of standardised indicators, as recommended and elaborated upon in the guidelines, to facilitate quality management and promote information exchange in the context of continuous quality improvement.

\section{Epilogue}

This chapter serves as an overview of the guidance available for CRC screening in the US, UK, Europe and Australia and briefly discussed the important role of GPs/FPs and PCPs, in general, in increasing the CRC screening rate. Although the literature is rich in information, guidelines and recommendation for CRC screening, there is room for improvement. It is to important invest in translating primary research into practice and combine qualitative and quantitative evidence for relevant, contextualised training and educational interventions, both at patient and provider levels.

\section{References and internet resources}

\subsection{References}

Allison JE, Sakoda LC, Levin TR, Tucker JP, Tekawa IS, Cuff T, Pauly MP, Shlager L, Palitz AM, Zhao WK, Schwartz JS, Ransohoff DF, Selby JV. (2007). Screening for colorectal neoplasms with new fecal occult blood tests: update on performance characteristics. J Natl Cancer Inst. Vol. 3;99(19):1462-70. Epub 2007, Sep 25.

Andermann, A., Blancquaert, I., Beauchamp, S., Déry, V.. (2008). Revisiting Wilson and Jungner in the genomic age: a review of screening criteria over the past 40 years. Bull World Health Organ. Vol. 86(4):317-9.

Aubin-Auger, I., Mercier, A., Lebeau. J.P., Baumann, L., Peremans, L., Van Royen, P. (2011). Obstacles to colorectal screening in general practice: a qualitative study of GPs and patients. Family Practice. Advance Access: cmr020 first published online May 6, 2011 doi:10.1093/fampra/cmr020.

Bampton, P.A., Sandford, J.J., Cole, S.R., Smith, A., Morcom, J., Cadd, B., Young, G.P. (2005). Interval faecal occult blood testing in a colonoscopy based screening programme detects additional pathology. Gut. Vol. 54(6):803-6.

Bastani, R., Yabroff, K.R., Myers, R.E. and Glenn, B. (2004). Interventions to improve followup of abnormal findings in cancer screening. Cancer. Vol. 101:1188-1200. 
Bowen D.J., Kreuter M., Spring B., Cofta-Woerpel L., Linnan L., Weiner D., Bakken S., (...), Fernandez M. (2009) How We Design Feasibility Studies. American Journal of Preventive Medicine. Vol. 36(5):452-457.

Brotons, C., Björkelund, C., Bulc, M., Ciurana, R., Godycki-Cwirko, M., Jurgova, E., Kloppe, P., Lionis, C., Mierzecki, A., Piñeiro, R., Pullerits, L., Sammut, M.R., Sheehan, M., Tataradze, R., Thireos, E.A., Vuchak, J.; EUROPREV network. (2005) Prevention and health promotion in clinical practice: the views of general practitioners in Europe. Prev Med. Vol. 40(5):595-601.

Cardarelli, R. (2010). The Role of Primary Care Providers in Cancer Screening. Primary Care Institute. (Accessed online November 10, 2011:

http://www.centerforcommunityhealth.org/Portals/14/Reports/PCPCancerBrief Final\%282\%29.pdf).

Christie, J., Itzkowitz, S., Lihau-Nkanza, I., Castillo, A., Redd, W., Jandorf, L. (2008). A randomized controlled trial using patient navigation to increase colonoscopy screening among low-income minorities. Journal of the National Medical Association. Vol. 100(3):278-284.

Christou, A., and Thompson, S.C. (2010). "How could the National Bowel Cancer Screening Program for Aboriginal people in Western Australia be improved?" Report to the WA Bowel Cancer Screening Implementation Committee, Department of Health, Western Australia and Combined Universities Centre for Rural Health.

Citarda, F., Tomaselli, G., Capocaccia, R., Barcherini, S., Crespi, M. (2001). The Italian Multicentre Study Group: Efficacy in standard clinical practice of colonoscopic polypectomy in reducing colorectal cancer incidence. GUT. Vol. 48:812-815

Cole, S.R., Young G.P., Byrne, D., Guy, J.R., and Morcom, J. (2002). Participation in screening for colorectal cancer based on a faecal occult blood test is improved by endorsement by the primary care practitioner. J Med Screen. Vol (9):147-152.

Edwards, B.K., Ward, E., Kohler, B.A., Eheman, C., Zauber, A.G., Anderson, R.N., Jemal, A., Schymura, M.J., Lansdorp-Vogelaar, I., Seeff, L.C., van Ballegooijen, M., Goede, S.L., Ries, L.A. (2010). Annual report to the nation on the status of cancer, 1975-2006, featuring colorectal cancer trends and impact of interventions (risk factors, screening, and treatment) to reduce future rates. Cancer. Vol. 1;116(3):54473.

Eide, T.J. (1991). Natural history of adenomas. World J Surgery. Vol. 15:3-6.

European Commission, Directorate General for Health and Consumers, EAHC - Executive Agency for Health and Consumers, World Health Organisation. (2011). European guidelines for quality assurance in colorectal cancer screening and diagnosis. (Accessed online September 10, 2011:

http:/ / bookshop.europa.eu/is-bin/INTERSHOP.enfinity/WFS/EU-BookshopSite/en_GB/-/EUR/ViewPublication-Start?PublicationKey=ND3210390).

Faivre, J., Dancourt, V., Lejeune, C., Tazi, M.A., Lamour, J., Gerard, D., Dassonville, F., Bonithon-Kopp, C. (2004). Reduction in colorectal cancer mortality by fecal occult blood screening in a French controlled study. Gastroenterology. Vol. 126(7):167480. 
Federici A, Giorgi Rossi P, Bartolozzi F, Farchi S, Borgia P \& Guasticchi G. (2005). Survey on colorectal cancer screening knowledge, attitudes, and practices of general practice physicians in Lazio, Italy. Prev Med. Vol(41):30-35.

Harris, D.M., Borsky, A.E., Stello, B. et al. (2010). Toolkit for the System Approach to Tracking and Increasing Screening for Public Health Improvement of Colorectal Cancer Intervention. AHRQ Publication No 11-0016.

Hassan, C., Benamouzig, R., Spada, C., Ponchon, T., Zullo, A., Saurin, J.C., Costamagna, G. (2011). Cost effectiveness and projected national impact of colorectal cancer screening in France. Endoscopy. Vol. 43(9):780-93. Epub 2011 May 27.

IARC (2005). Cervix Cancer Screening. IARC Handbooks of Cancer Prevention. Volume 10.

Jørgensen, O.D., Kronborg, O., Fenger, C. (2002). A randomised study of screening for colorectal cancer using faecal occult blood testing: results after 13 years and seven biennial screening rounds. Gut. Vol. 50(1):29-32.

Klabunde, C.N., Frame, P.S., Meadow, A., Jones, E., Nadel, M., Vernon, S.W. (2003). A national survey of primary care physicians' colorectal cancer screening recommendations and practices. Prev Med. Vol. 36(3):352-62.

Klabunde, C.N., Lanier, D., Nadel M.R, et al. (2009). Colorectal Cancer Screening by primary care physicians: recommendations and practices; 2006-2007. Am J Prev Med. Vol.(37):8-16.

Koo J.H., You B., Liu K., Athureliya M.D., Tang C.W., Redmond D.M., Connor S.J., Leong R. (2011). Colorectal cancer screening practices is influenced by ethnicity of medical practitioner and patient. J Gastroenterol Hepatol. doi: 10.1111/j.14401746.2011.06872.x. [Epub ahead of print]

Levin, B., Lieberman, D.A., McFarland, et al. (2008). Screening and surveillance for the early detection of colorectal cancer and adenomatous polyps, 2008: a joint guideline from the American Cancer Society, the US Multi-Society Task Force on Colorectal Cancer, and the American College of Radiology. CA Cancer J. Clin. Vol 58:130-160.

Lionis C, and Petelos E. (2011). Early detection of colorectal cancer: barriers to screening in the primary care setting. Family Practice. Vol 28(6):589-91.

Lionis, C. (2007). Colorectal cancer screening and the challenging role of general practitioner/family physician: an issue of quality. Quality in Primary Care. Vol. 15:129-131.

McFarland, E.G., Levin, B., Lieberman, D.A., Pickhart, P., Johnson, C.D., Glick, S.N., Brooks, D., Smith, R.A. (2008). Revised colorectal screening guidelines: Joint effort of the American Cancer Society. CA Cancer J Clin Vol 2008;58:160.

McGregor, S.E., Hilsden, R.J., Murray, A., Bryant, H.E. (2004) Colorectal cancer screening: practices and opinions of primary care physicians. Prev Med. Vol 39(2):279-85.

Meissner, H.I., Breen, N., Klabunde, C.N., Vernon, S.W. (2006). Patterns of colorectal cancer screening uptake among men and women in the United States. Cancer Epidemiol Biomarkers Prev. Vol 15(2):389-94.

Miles, A., Cockburn, J., Smith, R.A., Wardle J. (2004). A perspective from countries using organized screening programs. Cancer. Vol. 101(5):201-1213. 
Ornstein, M., Nemeth, L.S., Jenkins, P.G., Nietert, P.J. (2010). Colorectal cancer screening in Primary Care: Translating Research into Practice. Medical Care Vol 48: 900-906.

Pox, C., Schmiegel, W., Classen, M. (2007). Current status of screening colonoscopy in Europe and in the United States. Endoscopy 39:168-173.

Quirke, P., Cuvelier, C., Ensari, A., Glimelius, B., Laurberg, S., Ortiz, H., Piard, F., Punt, C.J., Glenthoj, A., Pennickx, F., Seymour, M., Valentini, V., Williams, G., Nagtegaal, J.D. (2010). Evidence-based medicine: the time has come to set standards for staging, Journal of Pathology. Vol. 221(4):357-360.

Renehan AG, Egger M, Saunders MP, O'Dwyer ST. (2002). Impact on survival of intensive follow up after curative resection for colorectal cancer: systematic review and metaanalysis of randomised trials. BMJ. Vol. 6;324(7341):813.

Rudy, D.R., Zdon, M.J. (2000). Update on Colorectal Cancer. Am Fam Physician Vol 61:175970,1773-4.

Sarfaty, M. (2006) How to Increase Colorectal Cancer Screening Rates in Practice: a Primary Care Clinician's Evidence-Based Toolbox and Guide. Atlanta, GA: The American Cancer Society, National Colorectal Cancer Roundtable and Thomas Jefferson University. (Accessed online November 10, 2011:

http://www.cancer.org/acs/groups/content/documents/document/acspc024588.pdf).

Sarfaty, M., Wender, R. (2007). How to increase colorectal cancer screening rates in practice. CA Cancer J Clin. Vol. 57(6):354-66.

Schroy, P.C. 3rd, Lal, S., Glick, J.T., Robinson, P.A., Zamor, P., Heeren, T.C. (2007). Patient preferences for colorectal cancer screening: how does stool DNA testing fare? Am J Manag Care. Vol. 13(7):393-400.

Schroy, P.C., Mylvaganam, S., Davidson, P. (2011). Provider perspectives on the utility of a colorectal cancer screening decision aid for facilitating shared decision-making. Health Expect September 8 [Epub ahead of print], doi:10.1111/j.13697625.2011.00730.x.

Senore, C., Malila, N., Minozzi, S., Armaroli, P. (2010). How to enhance physician and public acceptance and utilisation of colon cancer screening recommendations. Best Pract Res Clin Gastroenterol. Vol 24(4):509-20.

Sillars-Hardebol, A.H., Carvalho, B., van Engeland, M., Fijneman, R.J., Meijer, G.A. (2012). The adenoma hunt in colorectal cancer screening: defining the target. Journal of Pathology. Jan;226(1):1-6. doi: 10.1002/path.3012. Epub 2011 Nov.

Stern, M.A., Fendrick, A.M., McDonnell, W.M., Gunaratnam, N., Moseley, R., Chey, W.D. A randomized, controlled trial to assess a novel colorectal cancer screening strategy: the conversion strategy-a comparison of sequential sigmoidoscopy andcolonoscopy with immediate conversion from sigmoidoscopy to colonoscopy in patients with an abnormal screening sigmoidoscopy. Am J Gastroenterol. Vol. 95:2074-2079.

Stone, E.G., Morton, S.C., Hulscher, M.E., Maglione, M.A., Roth, E.A., Grimshaw, J.M., Mittman, B.S., Rubenstein, L.V., Rubenstein, L.Z., Shekelle, P.G. (2002). 
Interventions that increase use of adult immunization and cancer screening services: a meta-analysis. Ann Intern Med. Vol. 136:641-651.

Sung, J.J., Lau, J.Y., Goh, K.L., Leung, W.K.; Asia Pacific Working Group on Colorectal Cancer. (2005) Increasing incidence of colorectal cancer in Asia: implications for screening. Lancet Oncol. Vol 6(11):871-6.

Taskila T., Wilson S., Damery S., Roalfe A., Redman V., Ismail T., Hobbs R. (2009). Factors affecting attitudes toward colorectal cancer screening in the primary care population. Br J Cancer. Vol 21;101(2):250.

Tu S.P., Taylor V., Yasui Y, Chun A., Yip M.P., Acorda E. et al. (2006). Promoting culturally appropriate colorectal cancer screening through a health educator: a randomized controlled trial. Cancer. Vol 107:959-966.

Vernon SW. (2003). A national survey of primary care physicians' colorectal cancer screening recommendations and practices. Prev Med. Vol. 36(3):352-62.

Viguier J, Calazel-Benque A, Eisinger F, Pivot X. (2011). Organized colorectal cancer screening programmes: how to optimize efficiency among general practitioners. Eur J Cancer Prev. Vol. 20 Suppl 1:S26-32.

Wald N.J. (1994). Guidance on terminology. J Med Screen. 1: 76.

Walsh, J.M., Posner, S.F., Perez-Stable, E.J. (2002) Colon cancer screening in the ambulatory setting. Prev Med. Vol 35(3):209-18.

Wardle, J., Williamson, S., McCaffery, K. (2003). Increasing attendance at colorectal cancer screening: testing the efficacy of a mailed, psychoeducational intervention in a community sample of older adults. Health Psychology. Vol. 22:99-105.

Wilson, J.M.G., and Jungner, G. (1968). Principles and practice of screening for disease. Geneva: World Health Organization.(Accessed online October 25, 2011 from: http://www.who.int/bulletin/volumes/86/4/07-050112BP.pdf).

Winawer, S.J., Zauber, A.G., Ho, M.N., O'Brien, M.J., Gottlieb, L.S., Sternberg, S.S., Waye, J.D., Schapiro, M., Bond, J.H., and Panish,J.F. (1993). Prevention of colorectal cancer by colonoscopic polypectomy. The National Polyp Study Workgroup, New England Journal of Medicine. Vol. 329(27):1977-1981.

Young, G.P., St John, D.J., Winawer, S.J., Rozen, P. WHO (World Health Organization) \& OMED (World Organization for Digestive Endoscopy). (2002). Choice of faecal occult blood tests for colorectal cancer screening: recommendations based on performance characteristics in population studies: a WHO (World Health Organization) and OMED (World Organization for Digestive Endoscopy) report. Am J Gastroenterol. Vol 97(10):2499-507.

\subsection{Internet resources}

American Cancer Society (ACS) Guidelines for the early detection of cancer: Colorectal cancer and polyps.

(Accessed online September 19, 2011:

http://www.cancer.org/Healthy/FindCancerEarly/CancerScreeningGuidelines/a merican-cancer-society-guidelines-for-the-early-detection-of-cancer). 
Australian Government Department of Health and Ageing. (2008). Clinical Practice Guidelines for the Prevention, Early Detection and Management of Colorectal Cancer - A Guide for General Practitioners. (3rd ed.).

(Accessed online August 11, 2011:

http://www.health.gov.au/internet/screening/publishing.nsf/Content/bw-gpcrc-guide).

Australian Government Department of Health and Ageing. (2005) A Qualitative Evaluation of Opinions, Attitudes and Behaviours Influencing the Bowel Cancer Screening Pilot Program. Final Report. Screening Monograph: 2/2005.

(Accessed online September 16, 2011:

http://www.health.gov.au/internet/screening/publishing.nsf/Content/qualeval-cnt).

Australian Population Health Development Principal Committee. (2008). Population-Based Screening.

(Accessed online September 16, 2011:

http://www.cancerscreening.gov.au/internet/screening/publishing.nsf/Content /other-pop-health\#framework).

Cancer Research UK. Bowel cancer - screening and prevention.

(Accessed online September 15, 2011:

http://info.cancerresearchuk.org/cancerstats/types/bowel/screeningandpreventi on/).

Centers for Diseases Control and Prevention (US) MMWR. Vital Signs: Colorectal Cancer Screening, Incidence, and Mortality: United States, 2002-2010.

(Accessed online September 15, 2011:

http://www.cdc.gov/mmwr/preview/mmwrhtml/mm6026a4.htm?s_cid=mm602 $\left.6 a 4 \_w\right)$.

National Institute for Health and Clinical Excellence, (2011). NICE publishes new guidelines on colonoscopic surveillance for the prevention of colorectal cancer in people with ulcerative colitis, Crohn's disease or adenomas.

(Accessed online August 9, 2011:

http://www.nice.org.uk/newsroom/pressreleases/2011045colonoscopicsurveillan ce.jsp).

International Agency for Research on Cancer. Early detection and Prevention - Quality Assurance Group Resources.

(Accessed online August 11, 2011:

http://www.iarc.fr/en/researchs-groups/QAS/current-topics.php).

Patient-Centered Outcomes Research Institute. (2011-ongoing) Review and Synthesis of Evidence for Eliciting the Patient's Perspective in Patient-Centered Outcomes Research. Literature review and interviews.

(Accessed online September 19, 2011:

http:/ / www.pcori.org/patient-centered-outcomes-research/ and http://www.pcori.org/committee-charters/).

U.S. Preventive Services Task Force. (2008). Screening for Colorectal Cancer.

(Accessed online August 8, 2011: 
http://www.uspreventiveservicestaskforce.org/uspstf/uspscolo.htm).

World Health Organization. (2011). Screening for Colorectal Cancer.

(Accessed online August 1, 2011:

http://www.who.int/cancer/detection/colorectalcancer/en/). 


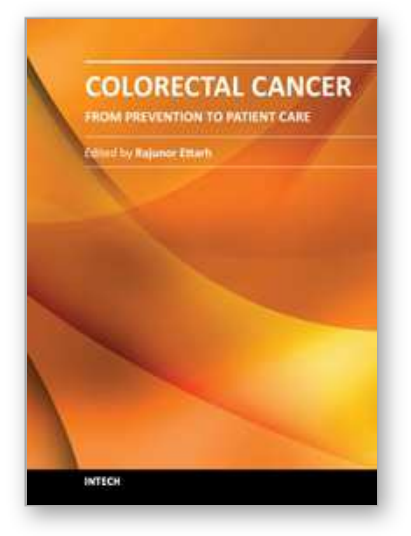

\author{
Colorectal Cancer - From Prevention to Patient Care \\ Edited by Dr. Rajunor Ettarh
}

ISBN 978-953-51-0028-7

Hard cover, 538 pages

Publisher InTech

Published online 17, February, 2012

Published in print edition February, 2012

The projections for future growth in the number of new patients with colorectal cancer in most parts of the world remain unfavorable. When we consider the substantial morbidity and mortality that accompanies the disease, the acute need for improvements and better solutions in patient care becomes evident. This volume, organized in five sections, represents a synopsis of the significant efforts from scientists, clinicians and investigators towards finding improvements in different patient care aspects including nutrition, diagnostic approaches, treatment strategies with the addition of some novel therapeutic approaches, and prevention. For scientists involved in investigations that explore fundamental cellular events in colorectal cancer, this volume provides a framework for translational integration of cell biological and clinical information. Clinicians as well as other healthcare professionals involved in patient management for colorectal cancer will find this volume useful.

\title{
How to reference
}

In order to correctly reference this scholarly work, feel free to copy and paste the following:

Christos Lionis and Elena Petelos (2012). Early Detection of Colorectal Cancer and Population Screening Tests, Colorectal Cancer - From Prevention to Patient Care, Dr. Rajunor Ettarh (Ed.), ISBN: 978-953-51-00287, InTech, Available from: http://www.intechopen.com/books/colorectal-cancer-from-prevention-to-patientcare/early-detection-of-colorectal-cancer-and-population-screening-tests

\section{INTECH}

open science | open minds

\author{
InTech Europe \\ University Campus STeP Ri \\ Slavka Krautzeka 83/A \\ 51000 Rijeka, Croatia \\ Phone: +385 (51) 770447 \\ Fax: +385 (51) 686166 \\ www.intechopen.com
}

\author{
InTech China \\ Unit 405, Office Block, Hotel Equatorial Shanghai \\ No.65, Yan An Road (West), Shanghai, 200040, China \\ 中国上海市延安西路65号上海国际贵都大饭店办公楼 405 单元 \\ Phone: +86-21-62489820 \\ Fax: $+86-21-62489821$
}


(C) 2012 The Author(s). Licensee IntechOpen. This is an open access article distributed under the terms of the Creative Commons Attribution 3.0 License, which permits unrestricted use, distribution, and reproduction in any medium, provided the original work is properly cited. 